\title{
Analysis of the fragile histidine triad (FHIT) gene in lobular breast cancer
}

\author{
C. Huiping a, J.G. Jonasson a , B.A. Agnarsson ${ }^{\text {a }}$, B.I. Sigbjornsdottir ${ }^{\text {a }}$, K. Huebner ${ }^{\text {b }}$, \\ S. Ingvarsson ${ }^{\mathrm{a}, *}$ \\ a Department of Pathology, University of Iceland, PO Box, 1465, IS-121 Reykjavik, Iceland \\ ${ }^{\mathrm{b}}$ Kimmel Cancer Center, Jefferson Medical College, Philadelphia, PA 19107-6799, USA
}

Received 20 December 1999; received in revised form 14 March 2000; accepted 23 March 2000

\begin{abstract}
The fragile histidine triad (FHIT) gene is a candidate tumour suppressor gene in breast and other cancers. We investigated deletions within the FHIT gene in lobular breast cancer and found that $16 \%$ of cases showed loss of heterozygosity (LOH) within the gene. We compared LOH within FHIT in lobular and ductal breast tumours and found a significant association between LOH at FHIT and the ductal histological type $(P<0.001)$. To determine whether genomic alteration of the FHIT gene in lobular breast cancer leads to Fhit inactivation we have assessed the level of Fhit expression by immunohistochemical detection and determined that 27\% (15 of 55) consecutive sporadic lobular tumours showed negative or reduced Fhit expression. A significant association was found between LOH at the FHIT gene and reduced Fhit expression in lobular and ductal tumours $(P=0.025$ and $P=0.001$, respectively). Thus, genetic alterations within the FHIT gene, leading to loss of Fhit protein, may play an important role in the carcinogenesis of a significant number of sporadic lobular breast cancers, even though the apparent frequency of genomic alterations within the gene is lower than in ductal breast cancer. (C) 2000 Published by Elsevier Science Ltd. All rights reserved.
\end{abstract}

Keywords: FHIT; Lobular breast cancer; LOH; Fhit expression

\section{Introduction}

The fragile histidine triad (FHIT) gene has been located at chromosome $3 \mathrm{p} 14.2$ [1]. It is a candidate tumour suppressor gene in breast and other cancers [24]. Genomic deletions within the FHIT locus have been observed in a variety of human cancers, including breast, lung, colon, oesophageal, head and neck, stomach, cervical and pancreatic carcinomas $[2,4]$. Frequent alterations of FHIT transcripts are also detected in some of these human cancer types [1,3,5-8]. The alterations of FHIT mRNA observed in tumours are often associated with the presence of genomic deletions within the FHIT locus in tumour-derived cell lines and tumour tissues [2]. Loss of heterozygosity $(\mathrm{LOH})$ studies indicate that more than $90 \%$ of lung tumours show LOH at the FHIT locus, and reverse transcriptase polymerase chain reaction (RT-PCR) analysis indicate

\footnotetext{
* Corresponding author. Fax: 3545601943.
}

E-mail address: siguring@rsp.is (S. Ingvarsson). that at least $80 \%$ of small cell lung cancers (SCLCs) and $40 \%$ of non-small cell lung cancers (NSCLCs) have alterations in the FHIT gene [5]. Moreover, $\mathrm{LOH}$ at the FHIT locus is higher in lung cancers of smokers than non-smokers [9], indicating that FHIT may be a target of the carcinogens present in tobacco smoke. To assess the frequency and consequence of FHIT rearrangements in human tumours, Siprashvili and colleagues [10] produced antiserum against the recombinant Fhit protein and used the antiserum to detect Fhit protein in tumour-derived cell lines and primary tumours. The results of this analysis indicated that tumour cell lines and tumours that exhibit altered FHIT transcripts and genomic FHIT alterations usually do not express or express reduced levels of Fhit proteins [7,8,11-13] reviewed in [4].

In sporadic breast cancer, LOH within the FHIT gene has been observed at different frequencies [14-16]. Similar deletions of the FHIT gene have been observed in preneoplastic lesions [14], suggesting that FHIT deletions could be an early event in a significant fraction of 
mammary carcinomas. Additionally, homozygous deletions within the FHIT gene have been observed in some breast cancer-derived cell lines [2,3,14], and $20-38 \%$ of primary breast carcinomas have been reported to exhibit altered FHIT transcripts $[3,11,17]$. Altered RT-PCR products are frequently due to internal deletions within FHIT, which appears to be inactivated by deletions rather than point mutations [4]. Furthermore, Bieche and colleagues [18] reported that in addition to a normal transcript, multiple variant transcripts were found at very low levels $(<1 \%$ compared with the amount of wild-type FHIT transcripts) in the majority of 33 breast tumours, but also in adjacent normal breast tissues and normal breast tissues from women without cancer. In a separate study, alterations in FHIT transcripts were detected in $30 \%$ of the patients, but a reduction or absence of Fhit protein occurred in $69 \%$ of the breast carcinoma samples examined [11].

In our previous studies, we located a smallest common deletion region (SCDR) at the 3 p14 region in sporadic breast tumours, and then we compared $\mathrm{LOH}$ at 3p14, in lobular and ductal breast tumours and found a significant association between $\mathrm{LOH}$ at 3 p14 and ductal histological type $[19,20]$. In this study, we analysed deletions using microsatellite markers located within the FHIT gene in lobular breast tumours and studied Fhit expression to understand whether alterations of the FHIT gene led to Fhit inactivation. We also compared lobular and ductal tumours to determine whether there is a histological difference in the involvement of the FHIT gene in tumorigenesis.

\section{Materials and methods}

\subsection{Patients and tumour material}

In all, 56 sporadic (all $B R C A 2$ 999del5 negative) and 5 BRCA2 999del5 lobular breast tumours were studied for $\mathrm{LOH}$ within the FHIT gene and expression of Fhit. Amongst the 56 sporadic lobular tumours, one was analysed for LOH only, 7 for expression of Fhit only and 48 for both. Primary breast carcinoma tissue was obtained on the day of surgery. Blood samples from the patients were collected in ethylene diamine tetra-acetic acid (EDTA) and if not processed immediately, tumours and blood were quickly frozen at $-70^{\circ} \mathrm{C}$. Oestrogen receptor (ER) status was recorded by the Department of Pathology of the University Hospital of Iceland. The blood samples were screened for the BRCA2 999del5 mutation that has been detected in $8.5 \%$ of Icelandic breast cancer patients [21]. In our earlier studies we have analysed the FHIT gene and the Fhit protein in BRCA2 999del5 tumours, and therefore, excluded those tumours of ductal origin in the present study [16]. We used 88 sporadic invasive ductal breast tumours for $\mathrm{LOH}$ and
Fhit staining analysis for comparison with lobular tumours, of which 3 were analysed for LOH only, 30 for expression of Fhit only and 55 for both. Fhit has been studied earlier in a proportion (27 cases) of the ductal breast cancer cases (16). Furthermore, we analysed the grade breakdown of 52 sporadic invasive ductal tumours, of which 13 were grade I, 18 grade II and 21 grade III.

\subsection{DNA isolation}

A salting out procedure [22] and a method described by Smith and associates [23] were used to obtain DNA from whole blood and tumour samples, respectively.

\subsection{Microsatellite marker analysis}

The PCR primers used for microsatellite marker analysis were localised at $3 \mathrm{p} 14.2$ within the FHIT gene: D3S4260 (intron 4), D3S1481 (intron 4), D3S2757 (intron 4), D3S1300 (intron 5) and D3S1234 (intron 5). DNA samples (25 ng) were subjected to PCR analysis in a total volume of $25 \mu$. DynaZyme ${ }^{\mathrm{TM}}$ polymerase (Finnzymes Oy, Espoo, Finland) was used in the buffer solution provided by the manufacturer with $100 \mu \mathrm{M}$ of each deoxynucleotide triphosphate and $0.25 \mu \mathrm{M}$ of primers. After $5 \mathrm{~min}$ of denaturation at $94^{\circ} \mathrm{C}$, samples were subjected to 35 cycles of amplification, consisting of $30 \mathrm{~s}$ at $94^{\circ} \mathrm{C}, 30 \mathrm{~s}$ at $55^{\circ} \mathrm{C}$ and $1 \mathrm{~min}$ at $72^{\circ} \mathrm{C}$, followed by a final extension for $10 \mathrm{~min}$ at $72^{\circ} \mathrm{C}$. The PCR products were denatured in formamide buffer, separated on $6.5 \%$ polyacrylamide sequencing gels, and transferred to a Hybond-N + Nylon membrane (Amersham, Aylesbury, UK). Hybridisation to the PCR products of a peroxidase-labelled probe was visualised using the ECL method (ECL kit, Amersham). LOH was evaluated visually by comparing the intensity of alleles from normal and tumour DNA. The absence or significant decrease in the intensity of one allele relative to the other was considered an allelic imbalance.

\subsection{Immunohistochemistry}

Fhit expression was analysed by Fhit antibody [10] staining of $5-\mu \mathrm{m}$ sections. The sections were deparaffinised, rehydrated, and rinsed in tap water before antigen retrieval by heating in a $0.01 \mathrm{M}$ citrate buffer (pH 6.0) twice for $5 \mathrm{~min}$ at $850 \mathrm{~W}$. Sections were incubated with the GST-Fhit antibody, diluted $1 / 800$, overnight at room temperature. Immunohistochemical staining was visualised using the Strept ABC Complex/ horseradish peroxidase (HRP) Duet (mouse/rabbit from DAKO) according to the manufacturer's instructions. Fhit expression in normal breast epithelial cells and stromal cells, respectively, served as positive and negative controls. Tumours were graded by intensity of 
staining as negative $(-)$, weakly positive $(+)$, moderately positive $(++)$ and strongly positive $(+++)$. The staining of 27 of the ductal breast cancers was based on tissue microarray and has been reported earlier [16].

\subsection{Statistical analysis}

A Chi-square test was used to assess the relationship between LOH within the FHIT gene in lobular and ductal breast tumours, between LOH at the FHIT gene in lobular and grade I ductal tumours and between Fhit expression in the lobular and ductal groups. A contingency analysis was also used to determine the association of Fhit expression in lobular and ductal groups. Then we analysed the association of $\mathrm{LOH}$ within the FHIT gene with Fhit expression in lobular and ductal tumours by Fisher's exact test and contingency analysis. Moreover, we detected the relationship between $\mathrm{LOH}$ at the FHIT gene and Fhit expression in all ER positive lobular tumours using the Chi-square test. A $P$ value of $<0.05$ was taken to be significant.

\section{Results}

In total, 49 sporadic lobular breast tumours were analysed for $\mathrm{LOH}$ using five microsatellite markers mapping to 3p14.2 within the FHIT gene. LOH with at least one marker was observed in 8 cases analysed $(16 \%)$ (Table 1). Fig. 1 is a $\mathrm{LOH}$ analysis showing the results from two tumours where breakpoints were detected within the FHIT gene, and Fig. 2 shows the results of eight lobular and eight ductal tumours showing $\mathrm{LOH}$ in comparison with Fhit expression. In most cases showing LOH with intragenic FHIT microsatellite markers, there is concordance among markers in the sense that $\mathrm{LOH}$ is detected at all informative loci in both lobular and ductal tumours.

Fifty-five lobular and 85 ductal breast cancer cases were examined for expression of Fhit by standard immunohistochemical detection using specific antisera for the protein (Fig. 3). A regional variation of the Fhit protein was detected across the tumours, but the scoring used here refers to the region of highest intensity of staining. In general, a stronger staining was detected in

Table 1

Deletions in the FHIT gene in lobular and ductal breast tumours ${ }^{\mathrm{a}}$

\begin{tabular}{lll}
\hline & \multicolumn{2}{c}{ No. of tumours } \\
\cline { 2 - 3 } FHIT & Lobular $n(\%)$ & Ductal $n(\%)$ \\
\hline LOH & $8(16)$ & $34(59)$ \\
ROH & $41(84)$ & $24(41)$ \\
\hline
\end{tabular}

$\mathrm{LOH}$, loss of heterozygosity; $\mathrm{ROH}$, retention of heterozygosity.

a In total, 49 lobular and 58 ductal tumours were analysed; $P<0.001$ by Chi-square test. the ductal compared with the lobular carcinomas. Because normal tissue is included in most of these sections, it served as an internal control and showed that normal epithelial cells are uniformly strongly positive for Fhit expression $(+++)$. Among the lobular tumours, $15(27 \%)$ cases were negative $(-)$ or weakly

(a)

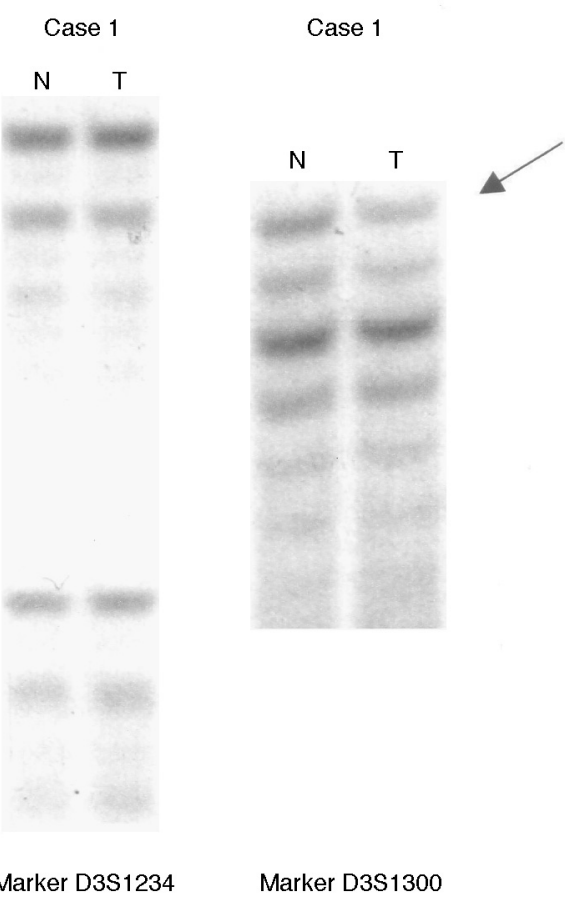

(b)

Case 5 Case 5

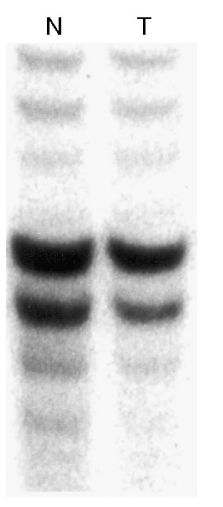

Marker D3S13

Fig. 1. Loss of heterozygosity (LOH) at the FHIT gene locus in matched normal $(\mathrm{N})$ and tumour $(\mathrm{T})$ tissues from cancer patients. (a) Tumour 1 shows retention of both alleles with marker D3S1234 but loss with marker D3S1300, (arrowed) and (b) tumour 5 shows retention with marker D3S1300 and loss with marker D3S4260 (arrowed). 


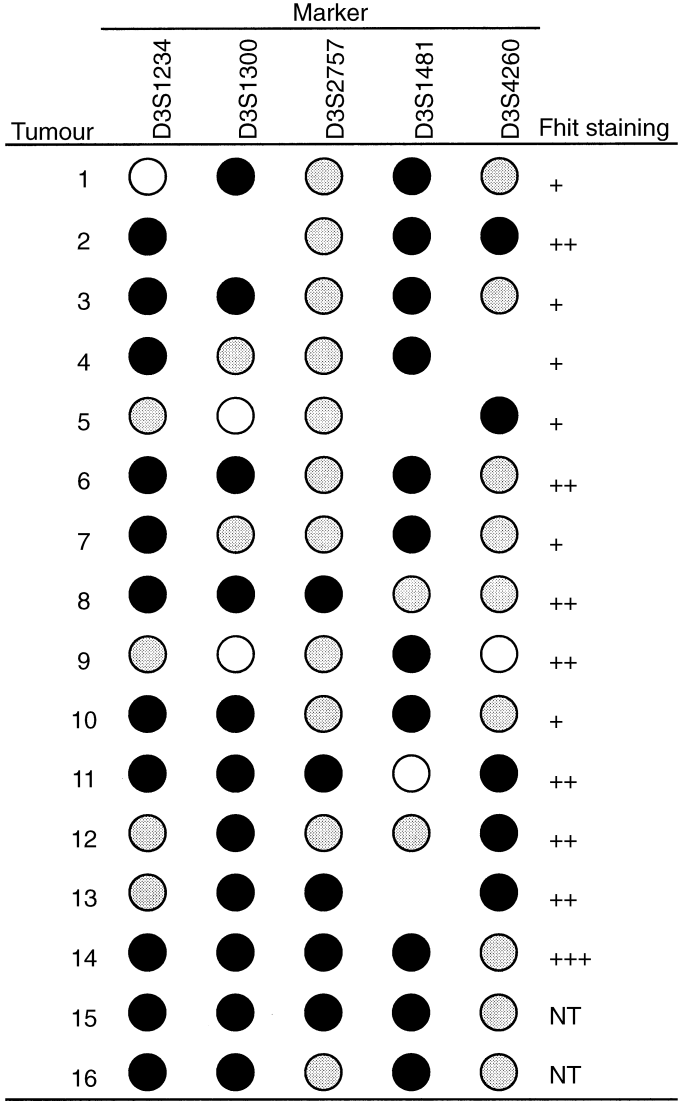

Fig. 2. Summary of loss of heterozygosity $(\mathrm{LOH})$ within the FHIT gene. Tumours 1-8 were lobular, and tumours 9-16 were ductal. $\bullet$, LOH; $\bigcirc$, homozygosity; $\bigcirc$, retention of heterozygosity. NT, no tumour material available for Fhit staining. Tumours 1, 5, 9 and 11 show breakpoints within the FHIT gene.

positive $(+), 30(55 \%)$ were moderately positive $(++)$ and $10(18 \%)$ were strongly positive $(+++)$ (Table 2$)$.

We compared LOH within the FHIT gene in lobular and ductal tumours and found a significant association between $\mathrm{LOH}$ at the FHIT gene and ductal histological type $(P<0.001)$ (Table 1$)$. All three grades of ductal tumours showed high LOH (grade I, 6/11 (55\%); grade II, 9/18 (50\%); grade III, $14 / 21(67 \%))$, a significant difference $(P=0.007,0.005$ and $<0.001$, respectively) in comparison with low LOH in lobular breast cancer in all cases. We also compared expression of Fhit in lobular and ductal tumours, but no significant difference was found (Table 2). To determine whether deletions within the FHIT gene led to a reduced expression of Fhit in lobular and ductal tumours, we compared the results from 48 lobular and 55 ductal cases, respectively, and found a significant association between $\mathrm{LOH}$ at the FHIT gene and reduced Fhit expression in the lobular and ductal tumours $(P=0.025$ and $P=0.001$ respectively) (Table 3). A majority (40/47) of the lobular breast tumours were ER-positive and we also found a significant association $(P=0.018)$ between $\mathrm{LOH}$ at the FHIT gene and reduced Fhit expression in this subgroup of tumours.
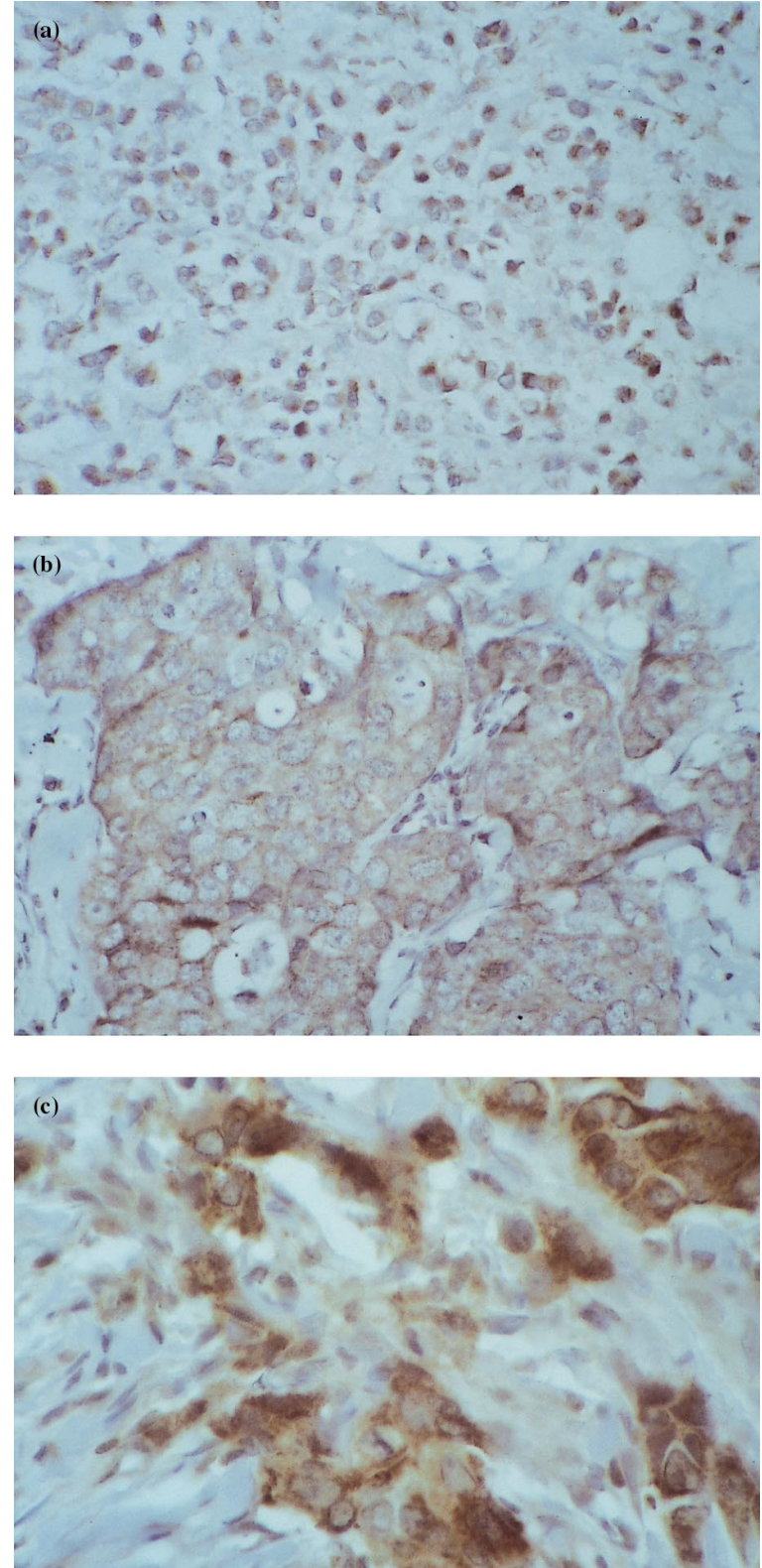

Fig. 3. Immunohistochemical detection of Fhit in (a) lobular (scoring + ); (b) ductal (scoring + ); and (c) ductal (scoring +++ ) breast cancers.

Table 2

Expression of Fhit in lobular and ductal breast tumours ${ }^{\mathrm{a}}$

\begin{tabular}{|c|c|c|}
\hline \multirow[b]{2}{*}{ Fhit expression } & \multicolumn{2}{|c|}{ No. of tumours } \\
\hline & Lobular $n(\%)$ & Ductal $n(\%)$ \\
\hline- & $2(4)$ & $4(5)$ \\
\hline+ & $13(24)$ & $22(26)$ \\
\hline++ & $30(55)$ & $28(33)$ \\
\hline+++ & $10(18)$ & $31(36)$ \\
\hline
\end{tabular}

a In all, 55 lobular and 85 ductal tumours were analysed; $P=0.052$ by contingency analysis. 
Table 3

Expression of Fhit in breast tumours with and without LOH at the FHIT gene $^{\mathrm{a}}$

\begin{tabular}{lllll}
\hline & \multicolumn{4}{c}{ No. of tumours } \\
\cline { 2 - 5 } & \multicolumn{2}{c}{ LOH (\%) } & \multicolumn{2}{c}{ ROH (\%) } \\
\cline { 2 - 5 } Fhit & Lobular & Ductal & Lobular & Ductal \\
expression & $n(\%)$ & $n(\%)$ & $n(\%)$ & $n(\%)$ \\
\hline- & 0 & $2(7)$ & $2(5)$ & $2(8)$ \\
+ & $5(63)$ & $16(52)$ & $6(15)$ & $1(4)$ \\
++ & $3(38)$ & $9(29)$ & $22(55)$ & $9(38)$ \\
+++ & 0 & $4(13)$ & $10(25)$ & $12(50)$
\end{tabular}

$\mathrm{LOH}$, loss of heterozygosity; $\mathrm{ROH}$, retention of heterozygosity.

a A total of 48 lobular and 55 ductal cases were assessed for both LOH at FHIT and Fhit expression. The difference in Fhit expression between tumours with $\mathrm{LOH}$ and tumours with $\mathrm{ROH}$ in lobular and ductal tumours was significant ( $P=0.025$ and $P=0.001$, respectively) by contingency analysis.

For $B R C A 2$ 999del5 lobular tumours, four of five showed LOH at the FHIT gene, three of five did not express Fhit protein, and three of five showed both LOH at FHIT and absence of Fhit expression.

\section{Discussion}

In this study, we found $16 \%$ of lobular breast tumours showed deletions within the FHIT gene, suggesting that alterations of the FHIT gene are involved in tumorigenesis in a subset of lobular breast tumours. However, higher LOH (59\%) within the FHIT gene was detected in ductal tumours than in lobular tumours $(P<0.001)$. This is consistent with our previous study [20], suggesting that deletions within the FHIT gene are frequent events in ductal breast tumours. Since both alleles are frequently altered in various cancers and since a family with hereditary cancer associated with a translocation disrupting one FHIT allele has been described, it is reasonable to consider FHIT a bona fide tumour suppressor gene [1,3]. To demonstrate suppressor activity, Siprashvili and associates have transfected the human FHIT cDNA into four different cell lines with homozygous deletions of the FHIT gene and then injected the Fhit-expression transfectants into nude mice, showing that the subsequent Fhit expression in these mice results in the loss of the ability to form tumours [10].

Fhit expression has been detected in both lobular and ductal tumours. We found that $27 \%$ of lobular cases and $31 \%$ of ductal cases showed negative or reduced Fhit expression, but this difference does not reach significant difference. It is consistent with our previous study that a proportion of sporadic breast tumours showed reduced expression of Fhit [16]. Some of these tumours are heterogeneous for Fhit expression. Fhit loss in preneoplastic bronchial lesions indicates the occurrence of genetic alterations associated with early steps of carcinogenesis [12]. Absence or reduction of Fhit expression in some breast and kidney neoplasias may accompany progression toward a more advanced stage of the disease $[11,13]$. Therefore, detection of Fhit expression by immunohistochemistry in premalignant and malignant tissues may provide important diagnostic and prognostic information and potential insights.

To determine whether there is an association of $\mathrm{LOH}$ at the FHIT gene with Fhit expression in both lobular and ductal tumours, we compared expression of Fhit in the cases with and without $\mathrm{LOH}$ within the FHIT gene in lobular and ductal tumours, respectively, and found a significant association between LOH at the FHIT gene and reduced Fhit expression in both tumour types. Our results suggest that FHIT could play an important role in lobular and ductal breast cancer due to loss of gene copy number, with a resultant loss of Fhit protein expression from the deleted allele. However, it should be noticed that the statistical analysis of lobular tumours was based on a low number of cases with $\mathrm{LOH}$ and reduced expression, since the majority of tumours did not show both loss of expression and $\mathrm{LOH}$. In addition, a relatively high number of lobular breast tumours $(8$ cases) lost protein expression and did not show $\mathrm{LOH}$. Abnormal expression control of the FHIT gene due to other mechanisms might be responsible for this discordance. Similarly, three lobular tumours out of eight showed FHIT LOH without Fhit protein loss. The different contribution of the two FHIT alleles to Fhit expression has not been analysed in this study but presumably some expression could occur from the remaining FHIT allele. In our previous study, based on a large panel of sporadic and $B R C A 2999 \mathrm{del} 5$ tumours that are mainly (approximately 90\%) of ductal histological type, we showed that there was a significant association of FHIT LOH and reduced expression of Fhit [16]. In addition, a heterogeneity in the expression of Fhit in subpopulations of tumour cells could possibly account for the lack of concordance between LOH at the FHIT gene and Fhit expression results in breast tumours. In this study, we detected high $\mathrm{LOH}$ at the FHIT locus and reduced Fhit expression in BRCA2 999del5 lobular breast tumours. These results are in line with our previous findings of FHIT abnormalities in BRCA2-linked ductal breast cancer [16].

In a previous study, we have shown that genetic alterations at the fragile site within the FHIT gene led to loss of Fhit expression in sporadic and familial breast tumours, and a larger fraction of familial cases with an inherited BRCA2 mutation showed FHIT alteration compared with sporadic cases [16]. In this study, we found that deletions within the FHIT gene led to reduced Fhit expression in a fraction of lobular breast tumours, suggesting that even though Fhit alterations 
occur at lower frequency compared with ductal breast cancer, they play a role in the pathogenesis of a significant proportion of lobular breast cancers.

\section{Acknowledgements}

The authors thank Sigrun Kristjansdottir and Kristrun Olafsdottir at the Department of Pathology, University Hospital of Iceland, for the immunohistochemical staining. This work was financially supported by the Icelandic Research Council and the Icelandic Cancer Society.

\section{References}

1. Ohta M, Inoue H, Cotticelli MG, et al. The FHIT gene, spanning the chromosome 3 p14.2 fragile site and renal carcinoma-associated $\mathrm{t}(3 ; 8)$ breakpoint, is abnormal in digestive tract cancers. Cell 1996, 84, 587-597.

2. Croce CM, Sozzi G, Huebner K. Role of FHIT in human cancer. J Clin Oncol 1999, 17, 1618-1624.

3. Negrini M, Monaco C, Vorechovsky I, et al. The FHIT gene at 3p14.2 is abnormal in breast carcinomas. Cancer Res 1996, 56, 3173-3179.

4. Huebner K, Garrison PN, Barnes LD, Croce CM. The role of the FHIT/FRA3B locus in cancer. Annu Rev Genet 1998, 32, 7 31.

5. Sozzi G, Veronese ML, Negrini M, et al. The FHIT gene at 3p14.2 is abnormal in lung cancer. Cell 1996, 85, 17-26.

6. Virgilio L, Shuster M, Gollin SM, et al. FHIT gene alterations in head and neck squamous cell carcinomas. Proc Natl Acad Sci USA 1996, 93, 9770-9775.

7. Greenspan DL, Connolly DC, Wu R, et al. Loss of FHIT expression in cervical carcinoma cell lines and primary tumors. Cancer Res 1997, 57, 4692-4698.

8. Simon B, Bartsch D, Barth P, et al. Frequent abnormalities of the putative tumor suppressor gene FHIT at 3 p14.2 in pancreatic carcinoma cell lines. Cancer Res 1998, 58, 1583-1587.

9. Sozzi G, Sard L, De Gregorio L, et al. Association between cigarette smoking and FHIT gene alterations in lung cancer. Cancer Res 1997, 57, 2121-2123.
10. Siprashvili Z, Sozzi G, Barnes LD, et al. Replacement of Fhit in cancer cells suppresses tumorigenicity. Proc Natl Acad Sci USA 1997, 94, 13771-13776.

11. Campiglio M, Pekarsky Y, Menard S, Tagliabue E, Pilotti S, Croce CM. FHIT loss of function in human primary breast cancer correlates with advanced stage of the disease. Cancer Res 1999, 59, 3866-3869.

12. Sozzi G, Pastorino U, Moiraghi L, et al. Loss of FHIT function in lung cancer and pre-invasive bronchial lesions. Cancer Res 1998, 58, 5032-5037.

13. Hadaczek P, Siprashvili Z, Markiewski M, et al. Absence or reduction of Fhit expression in most clear cell renal carcinomas. Cancer Res 1998, 58, 2946-2951.

14. Ahmadian M, Wistuba II, Fong KM, et al. Analysis of the FHIT gene and FRA3B region in sporadic breast cancer, preneoplastic lesions, and familial breast cancer probands. Cancer Res 1997, 57, 3664-3668.

15. Man S, Ellis IO, Sibbering M, Blarney RW, Brook JD. High levels of allele loss at the FHIT and ATM genes in non-comedo ductal carcinoma in situ and grade I tubular invasive breast cancers. Cancer Res 1996, 56, 5484-5489.

16. Ingvarsson S, Agnarsson BA, Sigbjornsdottir BI, et al. Reduced Fhit expression in sporadic and BRCA2-linked breast carcinomas. Cancer Res 1999, 59, 2682-2689.

17. Hayashi $\mathrm{S}$, Tanimoto K, Hajiro-Nakanishi $\mathrm{K}$, et al. Abnormal FHIT transcripts in human breast carcinomas: a clinicopathological and epidemiological analysis of 61 Japanese cases. Cancer Res 1997, 57, 1981-1985.

18. Bieche I, Latil A, Becette V, Lidereau R. Study of FHIT transcripts in normal and malignant breast tissue. Genes Chrom Cancer 1998, 23, 292-299.

19. Eiriksdottir G, Bergthorsson JT, Sigurdsson H, et al. Mapping of chromosome 3 alterations in human breast cancer using microsatellite PCR markers: correlation with clinical variables. Int $J$ Oncol 1995, 6, 369-379.

20. Huiping C, Sigurgeirsdottir JR, Jonasson JG, et al. Chromosome alterations and E-cadherin gene mutations in human lobular breast cancer. Br J Cancer 1999, 81, 1103-1110.

21. Johannesdottir G, Gudmundsson J, Bergthorsson JT, et al. High prevalence of the 999del5 mutation in Icelandic breast and ovarian cancer patients. Cancer Res 1996, 56, 3663-3665.

22. Miller SA, Dykes DD, Polesky HF. A simple salting out procedure for extracting DNA from human nucleated cells. Nucl Acids Res 1988, 16, 1215.

23. Smith SA, Easton DF, Evans DG, Ponder BA. Allele losses in the region 17q12-21 in familial breast and ovarian cancer involve the wild-type chromosome. Nat Genet 1992, 2, 128-131. 